\title{
Uptake and Perceptions of E-cigarette use in Vascular Patients
}

\author{
Rachael Taylor, David Sidloff, Robert D. Sayers, Matthew J. Bown, and Athanasios Saratzis \\ NIHR Leicester Cardiovascular Biomedical Research Unit, University of Leicester, Leicester, UK
}

\begin{abstract}
ntroduction: Smoking is one of the most important risk factors for cardiovascular disease (CVD). Electronic cigarettes (e-cigarettes) are becoming increasingly popular. However, little is known regarding their patterns of use in patients with established CVD.

Aims: We aimed to assess the perceptions and patterns of use of e-cigarettes in patients presenting to a vascular clinic.

Methods: We performed a qualitative study to identify perceptions and beliefs about e-cigarettes. Semi-structured interviews of consecutive patients consenting to participate were performed over fivemonths. Individuals were recruited from a vascular surgery outpatient clinic. Initial interviews were based on a questionnaire. Further structured interviews were conducted with patients currently using e-cigarettes, which were transcribed and analysed to assess perceptions and patterns of use.

Results/Findings: Four overarching themes emerged: attraction to e-cigarettes as a harm reduction/smoking cessation strategy; uncertainty regarding the risks of e-cigarettes; use of various types of smoking cessation strategies; dual use and often complete relapse to tobacco products.

Conclusions: Patients with established CVD view e-cigarettes as a means of smoking cessation; however, many relapse to tobacco products or use both simultaneously. Further research is necessary regarding the role of e-cigarettes in smoking cessation in this high-risk group.
\end{abstract}

\section{Introduction}

Smoking is one of the most important risk factors associated with cardiovascular and aortic disease (Katsiki, Papadopoulou, Fachantidou, \& Mikhailidis, 2013), responsible for 700,000 deaths annually in the European Union (Maurice, 2014) and causing hundreds of billions of dollars of economic harm annually in the form of excess healthcare costs and lost productivity. Smoking Cessation has therefore been prioritised by the World Health Organisation as part of disease prevention, and is a key strategy in managing patients with cardiovascular disease (CVD). A Cochrane systematic review found that smoking cessation was associated with a $36 \%$ reduction in the risk of long-term all-cause mortality in patients with established CVD (Critchley \& Capewell, 2004) whilst a large individual patient meta-analysis of data from the Consortium on Health and Ageing Network of Cohorts in Europe and the United States (CHANCES) consortium (Mons et al., 2015) demonstrated that smoking cessation reduces smoking related cardiovascular risk continuously over time.
Electronic cigarettes (e-cigarettes) are handheld electronic devices which vaporise a liquid typically consisting of nicotine, glycerine, various constituents and flavourings (McRobbie, Bullen, Hartmann-Boyce, \& Hajek, 2014) which the user subsequently inhales. This process is fundamentally different from smoking tobacco, and consequently the composition of the aerosol from e-cigarettes and the smoke from tobacco is likely to have different effects. The role of e-cigarettes as a smoking cessation aid remains unclear for example some data suggests that e-cigarettes may help smokers reduce their overall cigarette consumption (McRobbie et al., 2014) whilst other studies have demonstrated that vaping was associated with a higher risk of more frequent and heavy smoking six months later (Leventhal et al., 2016). Furthermore, habitual e-cigarette may be associated with increased cardiovascular risk (Benowitz \& Burbank, 2016; Moheimani et al., 2017) as although tobacco combustion causes most of the adverse health effects of smoking, nicotine which is present in e-cigarettes releases catecholamines (causing several hemodynamic effects) and has adverse effects on

Address for correspondence: Athanasios Saratzis MBBS MRCS FHEA PhD, NIHR Academic Clinical Lecturer - Vascular Surgery, Department of Cardiovascular Sciences \& NIHR Cardiovascular Biomedical Research Unit, University of Leicester, Leicester Royal Infirmary, LE15WW. Email: as875@le.ac.uk 
lipids, and inducing endothelial dysfunction and insulin resistance.

Public Health England have recently published an evidence update on e-cigarettes, commenting that they possess around $4 \%$ of the relative harm of cigarettes overall (including social harm) and 5\% of the harm to users themselves (England, 2014). The National Institute for Health and Care Excellence (NICE) does not currently provide any guidance regarding the use of e-cigarettes among patients with established CVD and there is a lack of research in this context, especially regarding their longterm cardiovascular impact (NICE, 2015). The main constituents in e-cigarette vapour of concern with respect to CVD are nicotine, carbonyls, and the various particulates. Long-term exposure to nicotine has been linked to CVD before via a plethora of mechanisms; however, the impact of the other substances remains largely unknown (Morris et al., 2015). Overall, there are definite concerns regarding the uptake of e-cigarettes among people who don't smoke and their long-term effect on CVD. Following the above, the aim of this study was to perform an exploratory analysis of patients with established CVD to assess e-cigarette uptake and a qualitative descriptive analysis to identify perceptions and beliefs about e-cigarettes in this high-risk patient group.

\section{Methods}

\section{Recruitment}

Between April and September 2016 (21-week period) all patients attending the general vascular surgery outpatients in a tertiary referral centre for vascular disease with a diagnosis of established peripheral arterial, aneurysmal or venous disease were contacted (in person) immediately after their clinic appointment. Patients were asked to participate in an initial short semi-structured qualitative interview (phase 1), following written informed consent. Patients were consented for future contact and interviewing if they were active smokers or e-cigarette users. Ethical approval was granted by the relevant institution.

\section{Interviews}

A member of the study team performed the initial clinic interview based on a standardised structured questionnaire (24 multiple choice questions), in order to assess their smoking habits and perceptions regarding e-cigarette use. A full medical history alongside basic demographic data were recorded and transferred onto an electronic bespoke database. Medical notes were accessed to obtain missing data where necessary. Using data from the initial semi-structured interviews, all of the e-cigarette users were then contacted at a later date (phase 2) in order to perform a subsequent telephone interview, which was recorded and transcribed verbatim. We aimed to assess patient perceptions regarding the use of e-cigarettes, using targeted questions to guide the conversation. All interviews were telephonic and were performed by a clinician (vascular surgeon). The participants were all asked about: why they chose to use e-cigarettes, duration and pattern of use, simultaneous use of tobacco-based products, perceived impact on health, activities, lifestyle and cost and use of other smoking cessation strategies.

\section{Analysis}

Following transcription (verbatim), the transcripts were analysed systematically using NVivo 11 Software (QSR International, Melbourne, Australia) and compared in order to identify cross-cutting themes and highlight common or differing experiences and views. Particular attention was paid to how participants categorised products, how they thought they should be used, how they talked about health risks, and whether/how they perceived e-cigarettes as a means of smoking cessation. The overarching themes were then discussed between co-authors to identify the most common opinions.

\section{Results \\ Phase 1}

Overall, 74 consecutive patients agreed to participate with none declining in the semi-structured interview phase based on a questionnaire. This took place in vascular outpatient clinics of a tertiary referral vascular unit, between April and September 2016. During that time-period a total of 1,682 eligible outpatients were seen in the institution. These semi-structured interviews took place immediately after attending a vascular clinic appointment. Medical and demographic data were prospectively collected and are reported on (Table 1). The majority of patients (48 patients - 65\%) were ex cigarette smokers with a mean quit length of 19 years (SD: 2 years). A total of nine patients were current e-cigarette users (12\%), of which the overwhelming majority (eight patients) had been tobacco smokers prior to their use.

Of the 74 patients who participated in the $1^{\text {st }}$ study phase (questionnaire), 11\% (eight individuals, of which six were already using an e-cigarette) reported that they viewed e-cigarettes as a tool that can help smoking cessation. Four of those eight participants (50\%) reported that they intended to stop smoking using an e-cigarette in the future (Table 2). (Table 2) summarizes findings of the $1^{\text {st }}$ phase (semi-structured interviews based on a questionnaire).

\section{Phase 2}

Following the above interviews, we identified nine individuals (two females, mean age: 67, SD: 12) who were currently using e-cigarettes. In this sub-group, the reasons for referral to the vascular clinic included: five patients with claudication, one with critical limb ischaemia, one with an asymptomatic abdominal aortic aneurysm and one with asymptomatic carotid artery disease. We conducted a further telephone interviews with these nine patients, after the initial semi-structured questionnaire 


\section{Table 1}

Patient medical and demographic data for the 74 qualitative interview participants (study phase 1)

\begin{tabular}{ll}
\hline Age & $69.2+/-$ \\
\hline Female Sex & $16(22 \%)$ \\
Diabetes & $11(14 \%)$ \\
Hypercholesterolaemia & $28(37 \%)$ \\
Hypertension & $33(45 \%)$ \\
CKD & $4(5 \%)$ \\
IHD & $12(16 \%)$ \\
CVA or TIA & $4(5 \%)$ \\
COPD & 0 \\
Asthma & 0 \\
Aspirin & $16(21 \%)$ \\
Clopidogrel & $8(11 \%)$ \\
Statin & $23(31 \%)$ \\
Diagnosis at vascular clinic appointment & \\
Claudication & 54 \\
Critical lower limb ischaemia & 2 \\
Abdominal aortic aneurysm & 4 \\
Carotid artery disease & 12 \\
Venous ulcer & 14 \\
\hline CKD: &
\end{tabular}

CKD: chronic kidney disease.

IHD: ischaemic heart disease.

CVA or TIA: cerebrovascular accident or transient ischaemic attack.

COPD: chronic obstructive pulmonary disorder.

based interview in clinic. The following overarching themes emerged from these interviews:

(a) Attraction to e-cigarettes as a harm reduction or smoking cessation strategy: eight of the nine patients (88\%) started using an e-cigarette as they believed it would help them stop smoking traditional tobacco products; however, two of those participants had stated in phase 1 (questionnaire study) that they no longer believed e-cigarettes will help them stop smoking altogether. The average length of time these patients had been using an e-cigarette for was 11 months (SD: 4) and during that time only two of the nine individuals had not used any other tobaccobased product (22\%). However, all nine individuals found that e-cigarettes either led them to stop smoking completely for at least 1 month or significantly reduced the number of cigarettes smoked per day. Participants were asked the following question during phase 2: "Do you believe you can stop using tobacco products completely within one year with the help of an e-cigarette?" and could reply "Yes, No, Not sure". Four of the nine individuals hoped that they would stop smoking any tobacco product within one year after the interview with the aid of the ecigarette. The remaining five were not sure whether

\section{Table 2}

Tobacco and e-cigarette use amongst the 74 participants

\begin{tabular}{|c|c|}
\hline \multicolumn{2}{|l|}{ Variable } \\
\hline Never smoked & $18 / 74(24 \%)$ \\
\hline Current tobacco smoker & $8 / 74(11 \%)$ \\
\hline Intention to quit smoking & $4 / 8(50 \%)$ \\
\hline $\begin{array}{l}\text { Intention to use E-cigarettes as a tool to quit } \\
\text { smoking }\end{array}$ & $4 / 8(50 \%)$ \\
\hline Ex-tobacco smoker & $48 / 74(65 \%)$ \\
\hline Average quit length & 19 years (SD: 2) \\
\hline Prior use of E-cigarettes to quit smoking & $8 / 48(17 \%)$ \\
\hline Used a tobacco product in the last 31 days & $7 / 74(9 \%)$ \\
\hline Pack year history & 17 (SD: 3) \\
\hline Average duration of daily tobacco use & 15 years (SD: 3 ) \\
\hline E-cigarette user & $9 / 74(12 \%)$ \\
\hline $\begin{array}{l}\text { Smoker prior to E-cigarette use (1 participant was } \\
\text { previously using other tobacco products) }\end{array}$ & $8 / 9(88 \%)$ \\
\hline Addiction scale to tobacco products* & 6 (SD: 1) \\
\hline Family insist on patient quitting & $18 / 74(24 \%)$ \\
\hline $\begin{array}{l}\text { Patient referred to smoking cessation service in } \\
\text { the past }\end{array}$ & $18 / 74(24 \%)$ \\
\hline $\begin{array}{l}\text { Patients prescribed nicotine medication in the } \\
\text { past }\end{array}$ & $3 / 74(4 \%)$ \\
\hline $\begin{array}{l}\text { Patient has received medical advice to quit } \\
\text { smoking }\end{array}$ & $56 / 74(76 \%)$ \\
\hline Other current smokers at home & $16 / 74(22 \%)$ \\
\hline Allowed to smoke in the house & $16 / 74(22 \%)$ \\
\hline
\end{tabular}

* Patients were asked how addicted they were to tobacco products when they were using them (or currently if they were active smokers) on a scale from 1 to 10.

SD: standard deviation.

the e-cigarette would help them stop smoking any tobacco product.

(b) Uncertainty regarding the risks of e-cigarettes: none of the participants was aware of any research regarding the impact of e-cigarettes on health and none had consulted a doctor regarding whether e-cigarettes were less harmful than tobacco products. Seven participants were of the opinion that e-cigarettes were less harmful than traditional tobacco products and two thought they were as harmful. All participants expressed that they were confused regarding the health effects of e-cigarettes and had experience of advertising claiming that e-cigarettes were less harmful than tobacco-based products. Interestingly, three participants expressed that e-cigarette cost was a significant factor that may affect their decision to continue using the in the future.

(c) Use of various types of smoking cessation strategies: eight of the nine participants had tried at least two different smoking cessation strategies (all eight had tried nicotine patches, seven had tried nicotine 
gums, five had tried nicotine tablets and one had tried acupuncture) in the last one year and three had been referred to at least one smoking cessation clinic.

(d) Dual use and often complete relapse to tobacco products: Only two of the nine individuals had not used any other tobacco based product (22\%) whilst using the e-cigarette. Interestingly, all of the nine individuals claimed smoked at least one traditional cigarette during the last year. Three (33\%) individuals in fact had completely relapsed to cigarettes at least once during the last year. All nine found it challenging to stay completely off cigarettes or other tobacco products whilst using the e-cigarette. There was unanimous agreement though that e-cigarette use does reduce the craving for traditional tobacco, especially in the morning.

\section{Discussion}

In this exploratory qualitative study, we attempted to identify the main perceptions and beliefs about ecigarettes in patients with established vascular disease treated by vascular surgeons. We performed structured interviews in two phases and found that about a tenth of the patients who were approached were actively using e-cigarettes; four overarching themes emerged regarding e-cigarette use attitudes: attraction to e-cigarettes as a harm reduction or smoking cessation strategy; uncertainty regarding the risks of e-cigarettes; use of various types of smoking cessation strategies; dual use and often complete relapse to tobacco products.

Despite large healthcare and social campaigns and a change in attitude over the latter part of the $20^{\text {th }}$ century in most western societies, smoking remains one of the most important risk factors associated with CVD (Katsiki et al., 2013). A Cochrane systematic review found that smoking cessation was associated with a $36 \%$ reduction in the risk of long-term all-cause mortality in patients with established CVD (Critchley \& Capewell, 2004) and all major healthcare advisory groups view smoking cessation as one of the primary strategies in the management of any patient with a vascular pathology. Subsequently, healthcare providers make great efforts to reduce the prevalence of smoking by investing significant funds in smoking cessation campaigns, community clinics, medication and education. The e-cigarette could potentially be a useful tool in the attempt to help a patient quit smoking, as it may reduce the craving for traditional tobacco products. No previous research, to the best of our knowledge, had assessed its role as a smoking cessation tool in high risk individuals with established vascular disease. Our research showed that these patients do indeed view e-cigarettes as a cessation tool and most expressed the opinion that it reduces tobacco craving. However, dual use was very common and so was complete relapse to tobacco.

Another overarching theme of importance is the uncertainty regarding the potential harms or benefits of e- cigarettes; this is echoed by previous reviews on the value and role of e-cigarettes (Morris et al., 2015). The latter are usually marketed with claims of health benefits compared with smoking traditional tobacco products (Klein et al., 2016; Pokhrel et al., 2016). Before discussing the risks and benefits of e-cigarettes it is important to identify the key steps in their function. They heat a nicotine solution to generate an aerosol that is inhaled without actual tobacco combustion; the aerosol contains nicotine, carbonyls and particulates, all of which can potentially impact upon respiratory and cardiovascular functions.

Nicotine enhances angiogenesis, may directly impact upon processes involved in the formation of an atheromatic plaque and in mouse model has been linked to the development of AAA (Wang et al., 2012). Furthermore, nicotine inhalation leads to increase of catecholamines, which may cause systolic and/or diastolic hypertension. Nicotine also has adverse effects on lipid metabolism and induces endothelial dysfunction and insulin resistance (Heeschen et al., 2001; Lee \& Cooke, 2012; Nordskog, Blixt, Morgan, Fields, \& Hellmann, 2003). In e-cigarettes users, nicotine has been shown to increase heart rate after overnight abstinence in a clinical/laboratory assessment (Vansickel, Weaver, \& Eissenberg, 2012). Further clinical assessments of e-cigarette users have shown an increase in diastolic blood pressure; however, their effect on myocardial perfusion was less pronounced compared to regular cigarettes (Farsalinos, Tsiapras, Kyrzopoulos, Savvopoulou, \& Voudris, 2014). It is also important to note that nicotine replacement therapy in the form of tablets or patches has long been recommended as means to help smoking cessation in patients with established CVD (Verbiest et al., 2017).

As far as the other compounds of the e-cigarette aerosol are concerned, the main substances that may have a potentially toxic effect are formaldehyde and acetaldehyde. The levels of these aldehydes in the e-cigarette aerosol is significantly lower than those in conventional cigarette smoke. Also, the risk of exposure to low levels of these substances remains unknown. Formaldehyde is a carcinogen, but its cardiovascular effects have not been investigated, especially over the long term. To the best of our knowledge, there are no large-scale epidemiological analyses assessing the impact of e-cigarette use on CVD either in the medium or short term. Also, we are not aware of any behavioural research investigating how individuals with CVD use e-cigarettes long-term and whether dual use (conventional tobacco products and ecigarettes used simultaneously) is indeed widely prevalent. Both these areas need to be researched further in the future.

\section{Limitations}

The main limitations of this study are the small number of participants and that recruitment was based at a single centre. However, the qualitative methods used do not 
allow large groups of patients to be interviewed; a focus group approach could potentially provide some further information in the future and that is an area of further research. Even though participants were recruited from a single centre, they were all diagnosed with common vascular pathologies that varied and represent the usual patient referred to a general vascular clinic. Future research in the field should attempt to elucidate the exact prevalence of e-cigarette usage in these patients, which may be possible using a different multi-centre approach. The long-term effects of e-cigarette smoking need also be assessed in high quality epidemiological studies.

\section{Conclusion}

E-cigarettes are a potentially valuable tool as part of an effective smoking cessation strategy in vascular patients. They should be viewed as part of a strategy that includes other established tools. Long-term data regarding their cardiovascular effects are awaited, hence prolonged use should not be encouraged.

\section{Acknowledgements}

Authors have no competing interests to declare and no funding specifically for this study has been granted.

\section{References}

Benowitz, N. L., \& Burbank, A. D. (2016). Cardiovascular toxicity of nicotine: Implications for electronic cigarette use. Trends in Cardiovascular Medicine, 26(6), 515-523.

Critchley, J., \& Capewell, S. (2004). Smoking cessation for the secondary prevention of coronary heart disease. Cochrane Database of Systematic Reviews, (1), CD003041.

England, P. H. (2014). E-cigarettes: An evidence update. Retrieved 12/12/2016, 2016, from https://www.gov.uk/ government/uploads/system/uploads/attachment_data/ file/457102/Ecigarettes_an_evidence_update_A_report_ commissioned_by_Public_Health_England_FINAL.pdf.

Farsalinos, K. E., Tsiapras, D., Kyrzopoulos, S., Savvopoulou, M., \& Voudris, V. (2014). Acute effects of using an electronic nicotine-delivery device (electronic cigarette) on myocardial function: Comparison with the effects of regular cigarettes. BMC Cardiovascular Disorders, 14, 78.

Heeschen, C., Jang, J. J., Weis, M., Pathak, A., Kaji, S., Hu, R. S. et al. (2001). Nicotine stimulates angiogenesis and promotes tumor growth and atherosclerosis. Nature Medicine, 7(7), 833-839.

Katsiki, N., Papadopoulou, S. K., Fachantidou, A. I., \& Mikhailidis, D. P. (2013). Smoking and vascular risk: Are all forms of smoking harmful to all types of vascular disease? Public Health, 127(5), 435-441.

Klein, E. G., Berman, M., Hemmerich, N., Carlson, C., Htut, S., \& Slater, M. (2016). Online E-cigarette marketing claims: A systematic content and legal analysis. Tobacco Regulatory Science, 2(3), 252-262.
Lee, J., \& Cooke, J. P. (2012). Nicotine and pathological angiogenesis. Life Sciences, 91(21-22), 1058-1064.

Leventhal, A. M., Stone, M. D., Andrabi, N., Barrington-Trimis, J., Strong, D. R., Sussman, S. et al. (2016). Association of e-Cigarette vaping and progression to heavier patterns of cigarette smoking. Journal of the American Medical Association, 316(18), 1918-1920.

Maurice, J. (2014). European union adds teeth to its antitobacco legislation. Lancet, 383(9920), 857-858.

McRobbie, H., Bullen, C., Hartmann-Boyce, J., \& Hajek, P. (2014). Electronic cigarettes for smoking cessation and reduction. Cochrane Database of Systematic Reviews, (12), CD010216.

Moheimani, R. S., Bhetraratana, M., Yin, F., Peters, K. M., Gornbein, J., Araujo, J. A. et al. (2017). Increased cardiac sympathetic activity and oxidative stress in habitual electronic cigarette users: Implications for cardiovascular risk. Journal of the American Medical Association Cardiology. https://www.ncbi.nlm.nih.gov/pubmed/28146259.

Mons, U., Muezzinler, A., Gellert, C., Schottker, B., Abnet, C. C., Bobak, M. et al. (2015). Impact of smoking and smoking cessation on cardiovascular events and mortality among older adults: Meta-analysis of individual participant data from prospective cohort studies of the .CHANCES consortium. British Medical Journal, 350, h1551.

Morris, P. B., Ference, B. A., Jahangir, E., Feldman, D. N., Ryan, J. J., Bahrami, H. et al. (2015). Cardiovascular effects of exposure to cigarette smoke and electronic cigarettes: Clinical perspectives from the prevention of cardiovascular disease section leadership council and early career councils of the american college of cardiology. Journal of the American College of Cardiology, 66(12), 1378-1391.

NICE. (2015). Smoking: Harm reduction. Retrieved 12/12/2016, 2016, from https://www.nice.org.uk/guidance/ qs92/chapter/Quality-statement-3-Advice-aboutnicotinecontaining-products.

Nordskog, B. K., Blixt, A. D., Morgan, W. T., Fields, W. R., \& Hellmann, G. M. (2003). Matrix-degrading and proinflammatory changes in human vascular endothelial cells exposed to cigarette smoke condensate. Cardiovascular Toxicology, 3(2), 101-117.

Pokhrel, P., Fagan, P., Herzog, T. A., Chen, Q., Muranaka, N., Kehl, L. et al. (2016). E-cigarette advertising exposure and implicit attitudes among young adult non-smokers. Drug and Alcohol Dependence, 163, 134-140.

Vansickel, A. R., Weaver, M. F., \& Eissenberg, T. (2012). Clinical laboratory assessment of the abuse liability of an electronic cigarette. Addiction, 107(8), 1493-1500.

Verbiest, M., Brakema, E., van der Kleij, R., Sheals, K., Allistone, G., Williams, S. et al. (2017). National guidelines for smoking cessation in primary care: A literature review and evidence analysis. NPJ Primary Care Respiratory Medicine, $27(1), 2$.

Wang, S., Zhang, C., Zhang, M., Liang, B., Zhu, H., Lee, J. et al. (2012). Activation of AMP-activated protein kinase alpha2 by nicotine instigates formation of abdominal aortic aneurysms in mice in vivo. Nature Medicine, 18(6), 902-910. 